\title{
La biomedicalización de la muerte: una revisión
}

\author{
Gemma Flores-Pons y Lupicinio Íñiguez-Rueda*
}

Universitat Autònoma de Barcelona

\begin{abstract}
Resumen: La biomedicina ha jugado un papel esencial en la definición de la muerte, estableciéndola como objeto del saber tecnocientífico a la vez que constituyéndola y performándola a partir de las prácticas biomédicas, sus equipamientos técnicos y sus espacios de acción. En el presente trabajo trazamos un recorrido por los principales temas de estudio que hemos identificado en los trabajos recientes alrededor de la biomedicalización de la muerte, como son (I) la buena muerte, (II) el ser en y para la muerte, (III) los cuidados paliativos, (IV) la muerte como decisión, (V) las prácticas de resucitación, (VI) la muerte tecnológica en las Unidades de Cuidados Intensivos (UCI) y (VII) la muerte encefálica. Por otra parte, también hacemos una rápida aproximación a los trabajos a partir de las perspectivas y tradiciones de investigación que están movilizando.

La muerte se presenta como un hecho controvertido en la sociedad actual y son múltiples los ámbitos médicos en los que se despliega como tal. Según cómo se defina qué es la muerte, se definirán los debates que la acompañan y viceversa, según cuáles sean las preocupaciones alrededor de la muerte, se delimitará en qué consiste ésta, en qué términos y con qué prácticas se puede definir.
\end{abstract}

Palabras clave: Muerte; medicalización; biomedicalización; medicina; biomedicina; tecnociencia.

\section{Introducción}

La medicina ha sido muy importante en la constitución del conocimiento de la persona, no sólo a nivel metodológico sino como disciplina constructora de realidad, ya que ha colocado al ser humano como objeto de saber a la vez que como sujeto poseedor de dicho saber. Se ha constituido como ciencia del individuo siendo portadora de formas concretas de existencia como es la salud y, a su vez, ha delineado esta existencia al regular y declarar la muerte (Foucault, 1963/1975). La biomedicina como tecnociencia, no trata la naturaleza del ser humano y se desarrolla gracias a un avance en el descubrimiento de la realidad sino que es un complejo proceso de co-construcción tanto de su objeto de estudio como de ella misma como disciplina. En otras palabras, la ontología del cuerpo que la biomedicina estudia se produce, no se descubre, en la interacción entre los instrumentos, los conocimientos, las personas y los haceres desarrollados en la práctica biomédica. La ontología del cuerpo y de aquellos fenómenos que la biomedicina estudia e interviene, devienen en la interacción, no la preceden. Son producto de las prácticas concretas en las que se encuentran insertos y que los actualizan (Latour, 1987/1992; Mol, 2002). Así, la biomedicina va desarrollándose y conformándose en el sí de esta dinámica de ajuste entre los diversos agentes que intervienen, como son el personal médico, las pacientes y sus afecciones, el entorno familiar, los afectos, los programas políticos, las directrices económicas del territorio o los conocimientos y las técnicas desarrolladas, entre otros (ver Rose, 2007).

* Dirección para correspondencia [Correspondence address]: Lupicinio Íñiguez-Rueda. Departament de Psicología Social. Universitat Autònoma de Barcelona. Edifici B. 08193 Bellaterra, Barcelona (España). E-mail: Lupicinio.Iniguez@uab.cat
Title: Biomedicalization of death: a review

Abstract: Biomedicine has played an essential role in death's definition by stablishing it as an object of technoscientific knowledge as well as constituting and performing it through biomedical practices, its technical equipments and its spaces of action. In this article we draw a route for recent works involving principal issues of study around the biomedicalización of death as are (I) the good death, (II) being in and for death, (III) palliative care, (IV) death as a decision, (V) resuscitation practices, (VI) the technological death in the Intensive Care Units (ICU) and (VII) brain death. Furthermore, we do a fast approach to the works through the research perspectives and traditions that they are mobilizing. Death is presented as a controverted fact in the existing society and are multiple fields of medicine in which it is spread as such. It results unmistakeable that according to how death is defined, debates that accompany it will be defined and vice versa, according to which are the worries about death, will be delimited what it is, in what terms and through which practices it can be defined.

Keywords: Death; medicalization; biomedicalization; medicine; biomedicine; technoscience.

Existe un acuerdo en que se ha dado un proceso por el cuál, en este caso, la muerte pasa a ser configurada por discursos y prácticas médicas, creándose un orden en el que la salud ocupa una posición central y la medicina deviene un régimen de verdad (Foucault, 1978/1980). Sin embargo, habrá autoras que definan este proceso en términos de medicalización como Banerjee (2008) mientras que otras optan por otros términos, como hace Holstein (1997) al hablar de colonización. Nosotras optamos por el término biomedicalización, entendiendo que la medicalización es un proceso dinámico que ha ido variando en el tiempo, intensificándose durante las últimas décadas alrededor de procesos de alta tecnificación de la medicina, adquiriendo especial relevancia para su desarrollo las ciencias bio-. Clarke, Mamo \& Fosket (2010) caracterizan estos cambios con la concreción de cinco procesos interactivos: (I) la expansión político-económica del sector biomédico; (II) la focalización en la salud, el riesgo y la vigilancia biomédica; (III) la tecnocientificación de las prácticas y las innovaciones en biomedicina; (IV) la transformación de los modos de producción, gestión, distribución y uso del conocimiento, (V) la transformación de los cuerpos, con nuevas propiedades, y la generación de nuevas identidades individuales y colectivas (Clarke, et al. 2010, p49). Cabe destacar que la biomedicina no actúa en la actualidad como una disciplina uniforme, por este motivo resulta útil aproximarse a la comprensión de su funcionamiento en la sociedad actual a partir de nuevas aportaciones como es la noción de plataformas biomédicas (Keating \& Cambrosio, $2000)^{2}$.

${ }^{2}$ Sirva de aclaración que, en el presente artículo optamos por los términos biomedicina y biomedicalización, y únicamente utilizaremos los términos medicina y medicalización cuando a) los trabajos citados los utilicen y b) nos refiramos a momentos históricos previos al "giro bio-". 
La muerte es un hecho sociohistóricamente situado que podemos comprender en su variabilidad no sólo como un objeto interpretable de formas diversas, sino como un objeto múltiple en sí mismo (Mol, 2002). Es decir, la imposibilidad de dar una definición singular de muerte se debe a que hay múltiples muertes que se encuentran parcialmente conectadas y esto es diferente a considerar que hay una sola muerte abordable desde una pluralidad de perspectivas y diferente también a considerar que hay una pluralidad de muertes no conectadas entre sí. En este sentido, la biomedicina ha jugado un papel esencial en su configuración, estableciéndola como objeto del saber tecnocientífico a la vez que constituyéndola y performándola a partir de las prácticas biomédicas, sus equipamientos técnicos y sus espacios de acción. Así, la muerte es un objeto cambiante que se ha ido articulando y performando al mismo tiempo que lo ha ido haciendo la biomedicina y la tecnociencia. Dicho en otros términos, la muerte habría ido pasando por procesos de juridificación y biomedicalización habrían obligado a una remodelación de los criterios que la definen (Rovaletti, 2002).

En este artículo presentamos una revisión bibliográfica sistemática de los estudios que se han realizado sobre la muerte como objeto biomédico. Hemos seleccionado aquellos trabajos recientes que de algún modo plantean una problematización o señalan alguna tensión en el hecho de definir la muerte como un objeto exclusivamente tecnocientífico. No obstante, consideramos que tanto aquellas prácticas que atienden a los aspectos tradicionalmente considerados sociales, como aquellas que atienden a los aspectos considerados biológicos, conforman las prácticas biomédicas actuales. De este modo, los trabajos centrados en definir los criterios tecnocientíficos de muerte que no plantean una problematización, a pesar de estar dando cuenta de la biomedicalización de la muerte, no han sido objeto de la revisión. Así, elaboramos una narración con la que ponemos de relieve, en primer lugar, algunos de los trabajos que actúan como referente en el estudio de la muerte en general. En segundo lugar, clasificamos las principales aportaciones de los trabajos más recientes alrededor de la muerte biomedicalizada. Con este objetivo, trazamos un recorrido por los trabajos revisados pasando por (I) la buena muerte, (II) el ser en y para la muerte, (III) los cuidados paliativos, (IV) la muerte como decisión, (V) las prácticas de resucitación, (VI) la muerte tecnológica en las Unidades de Cuidados Intensivos (UCI) y (VII) la muerte encefálica. Finalmente, hacemos una rápida aproximación a los trabajos a partir de las perspectivas y tradiciones de investigación en las que se sitúan como son la ética y la filosofía, la legalidad, la história y, por último, perspectivas y métodos psicosociales como la fenomenología, el análisis del discurso, la etnografía y la revisión bibliográfica sistemática.

\section{Método}

Para esta revisión hemos realizado una búsqueda bibliográfica general con la que hemos localizado los artículos publica- dos en revistas científicas durante las últimas décadas. Para ello, en las bases de datos y motores de búsqueda Dialnet, Directory of Open Access Journals (DOAJ), Jstor, Latinindex, Psycinfo, PubMed, Sage Journals Online, Sociological Abstracts, SpringerLink Journals, hemos cruzado las siguientes palabras clave:

- Español: muerte, muerte encefálica, muerte cerebral, cuerpo, corporalidad, hospital, UCI, salud, enfermedad, tecnología, ciencia, tecnociencia, medicina, medicalización, biomedicalización.

- Inglés: death, dying, brain death, cerebral death, body, embodiment, hospital, ICU, health, illness, technology, science, technoscience, medicine, medicalization, biomedicalization.

A partir de la lectura de los resúmenes, los títulos y las palabras clave, hemos seleccionado, guardado y clasificado, mediante el gestor de referencias bibliográficas Zotero 2.0.3, un total de 550 trabajos.

A continuación, hemos hecho un primer cribado de los estudios encontrados, priorizando aquellos que abordan la medicalización de la muerte y descartando aquellos que puedan resultar en el límite del tema o del abordaje. A continuación, hemos hecho varias relecturas de los abstracts, las palabras clave y los títulos y, con el programa Atlas.ti 5.6.3 (ver Muñoz-Justicia, 2005; Muñoz-Justicia \& Sahagún-Padilla, 2011), los hemos ido categorizando. Durante este proceso, hemos ido agrupando las categorías en famílias obteniendo la clasificación que presentamos en el presente artículo.

Con la lectura de los textos completos, hemos seleccionado, de cada categoría relevante, aquellos que resultan más destacables por su novedad, por la claridad de conceptos clave en el ámbito y por la diversidad metodológica y teórica. Además, estos trabajos seleccionados nos han servido como fuente para identificar, a partir de sus bibliografías, otros trabajos relevantes que no hemos encontrado con la búsqueda inicial en las bases de datos.

Finalmente, cabe destacar que, parte de los trabajos que exponemos aquí los hemos incorporado a través de otras prácticas propias del quehacer de la investigación como son la discusión de nuestro trabajo en seminarios y grupos de trabajo, las recomendaciones de compañeras y personas del entorno cercano y, finalmente, por las relevantes aportaciones realizadas por las personas que han evaluado el presente artículo.

\section{Resultados}

La muerte ha sido objeto de estudio desde múltiples disciplinas y ha dado pie a monográficos que la han examinado y analizado ya probablemente en todas sus formas. Algunos de ellos son relevantes y oportunos de mencionar, a pesar de no centrarse en el proceso concreto que en este artículo nos ocupa, la biomedicalización de la muerte.

Philippe Ariès, es uno de los autores básicos que han trabajado la muerte y lo ha hecho a partir de un análisis 
histórico que narró básicamente en los libros Historia de la muerte en Occidente $(1975 / 2000)$ y El hombre ante la muerte (1977/1987). A pesar de coincidir en muchos puntos, Norbert Elias en La soledad de los moribundos (1982/87), pone de relieve un cierto romanticismo presente en la obra de Ariès. Un romanticismo que dice impregnar su trabajo al transmitir el supuesto de que 'en épocas anteriores los hombres morían con serenidad y calma. Sólo en la actualidad, da Ariès por supuesto, han cambiado las cosas' (Elias, 1982, p.34). Extendiendo esta crítica, podemos decir que los análisis comparativos, tanto de tipo histórico como de tipo cultural, no quedan exentos de la dificultad de recoger la complejidad y la contradicción, dado que también Elias transmite un supuesto de linealidad y progreso cuando hace comparaciones entre las prácticas y vivencias alrededor de la muerte de diferentes culturas.

Para una extensa aproximación antropológica de la muerte, no puede faltar el trabajo de Louis-Vincent Thomas, principalmente desarrollado en el libro Antropología de la muerte (1975/1993) donde aborda las diferentes formas de morir, desde la muerte biológica hasta la muerte social y describe tanto las creencias como los rituales que establecen estas diferentes muertes. Igualmente, cabe destacar el libro La muerte (1991), de dimensiones mucho más reducidas que el anterior, en el que Thomas, evitando la exhaustividad y el detalle que tienen otros de sus trabajos, hace un ejercicio de síntesis y claridad para poner su abordaje de la muerte al alcance de toda aquella persona que esté interesada en generar una discusión alrededor de ésta sirviéndose de una lectura más ligera y esquemática.

Por último, para una revisión del estudio de la muerte en ciencias sociales, resulta clave la bibliografía elaborada por Marta Allué (1983) que supuso uno de los primeros pasos por establecer la muerte como un objeto a ser estudiado sistemáticamente y con continuidad.

La revisión bibliográfica que a continuación presentamos, la hemos elaborado principalmente a partir de nuestro interés por comprender, por un lado, cómo se construye la muerte en la actualidad y, por otro lado, las relaciones de poder que se producen con el desarrollo tecnocientífico, concretamente en el ámbito de la biomedicina. Frecuentemente, las controversias permiten aproximarse con mayor facilidad a aquellos objetos naturalizados en nuestra vida cotidiana y que hasta el momento han actuado de forma prácticamente aproblemática es decir, como cajas negras (Woolgar, 1988/1991). Por este motivo, en este trabajo nos centramos en aquellos trabajos que apuntan a tensiones alrededor de las prácticas biomédicas que constituyen la muerte.

Las categorías que presentamos a continuación no las hemos obtenido directamente sino que son el producto de agrupar los trabajos generando coherencia intracategorial y diferenciación intercategorial. Éste no es un proceso ni directo ni lineal sino que es el fruto de la constante toma de decisiones guiadas por los objetivos que ya hemos mencionado, así como por la voluntad de elaborar un artículo que pueda ser de utilidad para trabajos posteriores. Sin embargo, estas decisiones guardan información relevante, por este motivo queremos justificar aquí brevemente algunas de ellas, mientras que procuramos que otras queden apuntadas en cada uno de los apartados.

Los trabajos revisados presentan muchos puntos en común, sin embargo, cada uno suele enfatizar o profundizar más en un tema o a hacer una aportación novedosa en alguno en particular. De este modo, a pesar de que algunos artículos los categorizamos en los primeros análisis con varias de las categorías, finalmente la mayoría de ellos los adjudicamos a alguna en particular. Sin embargo, no se trata sólo de categorizar sino de narrar una revisión del contenido y de la relevancia de los trabajos y, por lo tanto, hemos considerado que algunos trabajos pueden requerir su mención en más de una categoría.

Un bloque significativo de los trabajos revisados gira alrededor de la noción de (I) la buena muerte, pero hemos procurado mantener bajo esta categoría sólo aquellos que la abordan de forma global y hemos mantenido categorías más específicas para aquellos trabajos que se refieren a ésta desde la particularidad de prácticas como son (II) el ser en y para la muerte, (III) la muerte como decisión y (IV) los cuidados paliativos. Esta última categoría, de hecho, hace de puente con el segundo bloque de trabajos, el cual recoge aquellos estudios centrados en las prácticas técnicas biomédicas que producen nuevos estados y nuevas formas de morir. Éstas son (V) las prácticas de resucitación, (VI) la muerte tecnológica en las Unidades de Cuidados Intensivos, en tanto que escenarios principales de las muertes actualmente $\mathrm{y}$, por último, (VII) la muerte encefálica, como caso de muerte tecnológica en las UCIs alrededor de la cual se ha generado un corpus importante de trabajos que, además, forma parte de nuestros objetivos de investigación actuales.

\section{La buena muerte}

La noción de buena muerte tiene un largo recorrido que Hart, Sainsbury \& Short (1998) y McNamara, Waddell \& Colvin (1994) resiguen y discuten de forma muy esclarecedora. Su significado actualmente resulta bastante amplio y cubre diferentes visiones, y podríamos decir que ha promovido, otras nociones que buscan una mayor especificidad como son las diferentes formas de eutanasia (ver Bayés, 2005; Gascón, 2003), los cuidados paliativos (ver Cannaerts, Dierckx \& Grypdonck, 2004), o el suicido asistido (ver Rosenfeld, 2004). Para el desarrollo y reivindicación de todas estas formas de aproximarse al final de la vida, ha sido fundamental el trabajo de Elizabeth Kübler-Ross (1969/2009), quién desarrolló un modelo de 5 estadios (modelo DABDA) por los que, afirma, pasaría la persona que va a morir. Cabe destacar que, su trabajo no ha estado exento de cuestionamientos (ver Friedman \& James, 2008) a pesar de reconocérsele un rol clave en la crítica de la práctica biomédica al final de la vida, así como en la generación de nuevas formas de hacer. 
Hart et al. (1998) defienden que, desde la edad media, la muerte ha pasado de ser un fenómeno natural o salvaje que era conocido y gestionado por la propia persona a partir de la celebración de rituales, la concesión de bendiciones y de perdón, a ser una cuestión controlada institucionalmente que produce formas aceptables de morir. Haciendo una revisión histórica, estos autores defienden que el concepto de buena muerte tiene carácter ideológico y analizan cómo su paso de ser reivindicación minoritaria a ser una ideología dominante dentro del hospital y en la sociedad en general resulta beneficioso a la vez que limitante. El hecho de reivindicar una mayor humanización de la muerte y el requerimiento de un cuidado, no sólo ha abierto nuevas posibilidades para el fin de la vida, sino que también ha normativizado qué es la buena muerte con su consecuente penalización y desaprobación de aquellas muertes y personas que se desvían de esta normalidad.

En este sentido, McNamara et al. (1994) concluyen que la buena muerte construye los roles de las personas cuidadoras así como de las pacientes, todo ello intentando encajar en la ideología del hospital marco. De este modo, aquellas muertes que encajen con la normalidad serán un éxito del sistema en el que se ha producido. Del mismo modo, aquellas muertes inadecuadas serán referidas como consecuencia de la singularidad de la persona o su entorno familiar. Así, a pesar de que creen que se está transformando la forma de concebir la buena muerte y tendiendo a contemplar mayor autonomía de la persona (McNamara et al., 1994; Volker \& Limerick, 2007), la transformación no está exenta de dificultades para superar el discurso dominante e instituído que da forma a las opciones actuales, las cuales proporciona y limita a la vez (Hart et al., 1998; McNamara et al., 1994; Proulx \& Jacelon, 2004).

Considerar que las muertes se producen a partir de procesos de normativización, tal y como lo plantean los estudios mencionados, conlleva a una cierta visión de uniformización que Long (2004) rehúsa al exponer que en las sociedades post-industriales no hay formas únicas de morir bien. A partir de un estudio etnográfico sobre los guiones culturales (cultural scripts) de la buena muerte en Estados Unidos y en Japón, la autora defiende que no hay diferencias que puedan definir una buena muerte típicamente Americana y una buena muerte típicamente Japonesa, sino que ambas tienen múltiples guiones, partes de los cuales comparten y partes en las que difieren. Asimismo, la autora apela, desde la perspectiva de la hermenéutica, a la creatividad de las personas a la hora de generar nuevos significados y nuevas formas de morir bien que se salen de los guiones preestablecidos, poniendo en juego lo que serían las macroestructuras y las interacciones micro.

El ámbito de la oncología ha generado también interesantes trabajos alrededor de la buena muerte, como Volker \& Limerick (2007) que han estudiado cómo conciben una muerte digna profesionales de enfermería en oncología. Cabe destacar que la noción de muerte digna, recoge en gran parte el legado de la buena muerte pero pretendería desmar- carse del juicio moral que supondría calificar de buenas y malas las muertes. Con la noción de dignidad se rescatan la autonomía y la particularidad de cada persona (ver Asociación Derecho a Morir Dignamente). Volker \& Limerick (2007) concluyen que se considera la integridad corporal y la paz en el momento de morir como factores generales, a la vez que incorporan otra dimensión que tiene en cuenta la especificidad de cada paciente, al considerar que una muerte digna debe contemplar los criterios propios de la persona que está por morir. También a partir de profesionales de enfermería en oncología, Volker (2001) ha abordado la experiencia de este sector ante la petición de muerte asistida, concluyendo que supone una situación conflictiva en la que el control y la comunicación juegan un papel fundamental aunque, en cualquier caso, se trata de experiencias que marcan a las personas y que perduran en su memoria.

\section{Ser en y para la muerte}

La muerte también ha sido abordada desde un interés por los roles en el proceso de morir y su encaje como acción microsocial en una estructura macrosocial. Emanuel, Bennett \& Richardson, (2007) defienden que habría unos roles disponibles a la hora de morir que vendrían determinados por una parte instintiva y otra social, pero especifican que algunos de estos roles tendrían una mayor acogida cultural que otros. Habría barreras actuando que impedirían el desarrollo del rol del moribundo (dying role), siendo éstas la falta de experiencia con la muerte, barreras culturales, dificultad de acceso a cuidados paliativos, un sistema de control de síntomas pobre, conocimiento inadecuado y otras barreras situacionales. Además, apuntan que estos roles podrían ser mal utilizados haciendo de ellos una norma, un uso prematuro, una imposición o un fracaso en su desarrollo por un desajuste con los roles de las personas acompañantes. En el trabajo citado, el personal médico se presenta como una pieza clave para el éxito del desarrollo del rol ya que puede tener control en gran parte de las barreras habituales.

A partir de un análisis conversacional, Li \& Arber (2006) estudian cómo se construye la credibilidad de estos pacientes por parte del personal de cuidados paliativos y cómo, a partir de la conversación, se están realizando acciones, no sólo compartiendo significados. Proulx \& Jacelon (2004) arguyen que la sociedad efectúa presión para que la persona que está muriendo ejerza de buena paciente. En este sentido, Banerjee (2008) utiliza la noción de subjetividad para comprender como el individuo es construido en y para la muerte. Así, llama subjetividades patológicas mortales a la forma como las personas nos construimos respecto la muerte: vivimos como seres mortales, experimentamos la muerte como una enfermedad y la enfrentamos como una batalla a ganar. Si bien esto tendría el beneficio de alargar, frecuentemente, la vida, también tendría efectos perversos como la consideración de la prolongación de la vida como un bien superior a la propia voluntad y experiencia de la persona. Asimismo se produce una mecanización del cuerpo y una tecnificación de 
los procesos de salud, enfermedad o muerte, de forma que otras dimensiones de la persona quedarían en desconsideración.

\section{La muerte como decisión}

La toma de decisiones, es una acción mediada que, lejos de ser un acto solipsista se produce distribuida en un entramado de relaciones del que participan múltiples agentes (Flores-Pons, Moreno, Íñiguez-Rueda \& Sanz, en prensa). En biomedicina, existe una tendencia a, ante la duda, optar por la vida pero también a borrar todo rastro de dicha duda, construyendo ciertas cosas como hechos y no decisiones y dejando la etiqueta de decisión sólo para ciertas opciones y ciertas situaciones. En los trabajos que sintetizamos aquí, se puede observar que una de las principales cuestiones en juego es que forma de plantear la muerte establece quién está legitimada para abordarla, definirla y decidir sobre ésta, como vemos en Bellomo \& Zamperetti (2007) o enPalmer (2000) que analiza concretamente el papel del personal médico y de enfermería, así como el rol de las leyes vigentes en la decisión.

Desde la sociología del conocimiento, Anspach (1987) aborda la toma de decisiones alrededor de la muerte y señala que los hospitales y, concretamente, las UCIs como espacios organizacionales producen experiencias y conocimientos diferentes entre el personal médico y el de enfermería, promoviéndose pronósticos y decisiones dispares dada la contingencia. En cambio, la incomprensión es uno de los principales problemas en la toma de decisiones alrededor de la muerte para Heyland, Tranmer \& Feldman-Stewart (2000) y añaden que puede solucionarse mejorando la comunicación. Para ello proponen un marco de comprensión para la toma de decisiones sobre la muerte basado en tres pasos analíticos: el intercambio de información, la deliberación y la toma de decisión. Buzzi (2009) cuestiona el carácter orientado a la acción de la medicina y de la forma de preguntar sobre la muerte. En contraposición, el autor prioriza conocer qué se entiende por 'persona' y 'vida' antes de poder abordar 'cómo actuar' respecto la enfermedad y la muerte.

Finalmente, nos parece importante el trabajo de Sharp (2006) quién advierte del riesgo de considerar ignorantes o supersticiosas aquellas decisiones contrarias a la propia perspectiva de una misma. Así, propone no uniformizarlas ni verlas como obstructivas sino como formas creativas de entender la muerte, aunque sean subversivas e impliquen un proceso biosocial complejo.

\section{Los cuidados paliativos}

La razón de ser de los cuidados paliativos se encuentra en medio de la dicotomía vida-muerte. Así, mientras que hay aproximaciones que los defienden como una gestión de la muerte, como es el caso de Taylor, Glass, McFarlane \& Stirling (1997), hay otros trabajos como el de Cannaerts et al. (2004) que consideran que el centro de los cuidados paliati- vos está en la calidad de vida. En la práctica, esto último implica conseguir que la enfermedad y la muerte no sean el centro sino que lo sea la vida y que las personas puedan disfrutarla incluso estando cerca de la muerte. Sin embargo, romper con la negación de la muerte no es suficiente para hacer que ésta sea agradable, sino que el sufrimiento también debe ser abordado, aunque sin considerar que pueda ser eliminado (Cannaerts et al. 2004; Zimmerman \& Rodin, 2004). Este sufrimiento no puede ser solamente abordado en términos físicos, sino que debe considerarse cómo la muerte es integrada en la vida cotidiana de la persona y su entorno. McPherson, Wilson \& Murray (2007) señalan que la sensación de ser una carga para las personas cercanas es una de las principales fuentes de sufrimiento, ya que se considera una pérdida de dignidad y en definitiva una mala muerte. Para Holstein (1997), la medicina ha colonizado la muerte y la ha transformado en un campo de batalla dónde el progreso aparece como ilimitado, a la vez que ha promovido la idea de que se puede hacer desaparecer la muerte como condición del ser humano. Por este motivo, destaca el potencial de los cuidados paliativos para reivindicar en medicina el mismo respeto por el cuidado que por la curación.

Por último, Menezes (2008) recoge, a partir de un trabajo etnográfico, cómo se produce la muerte en un hospital de cuidados paliativos y reporta que los cuidados paliativos resitúan la muerte como algo sobre lo cuál las personas pueden decidir y que mediante éstos se puede obtener la buena muerte. De este modo, describe cómo se interrelacionan en la práctica cotidiana los diferentes ejes de trabajo que hemos clasificado hasta el momento.

\section{Las prácticas de resucitación}

La práctica de la resucitación supone beneficios y riesgos tanto para el entorno familiar como para el equipo médico y esto suele valorarse a la hora de tomar la decisión de permitir la presencia de los familiares en el momento de la resucitación, tal y como explican McClement, Fallis \& Pereira (2009). Timmermans (1998) apunta a uno de los aspectos clave en todos los procesos de gestión biomédica de la muerte, especialmente en las Unidades de Cuidados Intensivos y no sólo en las prácticas de resucitación. Se trata de la conformación de un ritual que performa la confianza de las personas en el personal médico, en tanto que éste muestra haber hecho todo lo posible para salvar la vida de la persona. En otro trabajo, Timmermans \& Berg (1997) concluyen que el impacto de la presencia de familiares en el momento de resucituación se debe al paso de una situación técnica a una situación comunitaria. La complejidad de este paso reside en que, por un lado, la presencia de familiares no puede limitarse a una atestiguación del proceso sino que requiere de profesionales que den apoyo y, por otro lado, también debe tomarse en consideración cómo esta presencia interfiere en el escenario técnico de intervención que se produce actualmente en esta situación. Hadders (2007a, 2009a) y McGaheyOakland et al. (2007) remarcan que uno de los elementos 
más reclamados para abordar esta situación es la creación de protocolos que estandaricen y guíen esta práctica, y que recojan la diversidad de elementos mencionados.

\section{La muerte tecnológica en las unidades de cuidados intensivos (UCI)}

La tecnología tiene, actualmente, un relevante papel en la configuración de la muerte ya que es dentro de un entramado de relaciones económicas, políticas y culturales donde se producen ciertos desarrollos tecnocientíficos que permiten que aparezcan y se transformen los modos de morir (Kaufman, 2000). Turner (2004) explora qué principios éticos, políticos, económicos y sociales están promoviendo que la extensión de la vida se presente como deseable y destaca una creciente tendencia hacia esta perlongación. Es un tema que se ha convertido en el foco de la ciencia, las políticas públicas y la bioética, aunque debe destacarse que no tiene porque ser indicador de una mejora de la salud de la población (Olshansky et al., 1991). La muerte, dice Harvey (1997), en tanto que se produce eminentemente a partir de un marco médico, se encuentra altamente tecnificada, sin embargo esta tecnificación también busca emular la llamada muerte natural, intentando que no se produzcan muertes abruptas sino que sea un proceso gradual que la haga menos traumática.

A menudo, los estudios sociales señalan la tecnociencia como responsable de la aparición de muertes indignas (Timmermans, 1998). Jerez \& Rodriguez (1994) describen cómo la introducción de nuevas tecnologías biomédicas en la dinámica social se hace a partir de negociaciones y tensiones entre distintos actores y grupos sociales. En este marco, Hadders (2007b) se cuestiona cómo se puede compatibilizar una muerte digna con el cuidado intensivo altamente tecnificado y a menudo dramático que se produce en las UCIs. Estamos en un momento de negación de la muerte que pone mucho énfasis en la curación y en la creencia del progreso tecnocientífico como un incuestionable progreso social. Trabajos como los de Maynard (2006) o McGibbon \& Peter (2008) exponen la aparición de nuevas tecnologías como una interferencia constante en los dispositivos sociales de normalización que ejerce presión sobre las personas.

Las UCI son uno de los dispositivos más importantes actualmente en el cuidado en el fin de la vida. Seymour (2000) defiende que debido a la elevada tecnificación en este contexto y en la sociedad en general, hay un poco de confusión sobre las habituales dicotomías 'natural' y 'artificial'. Esta confusión se expresaría en prácticas como la extendida tendencia a utilizar soporte vital multiorgánico en casos en que se conoce la inevitabilidad de la muerte. Este conflicto se ve también alimentado por las posibilidades económicas y recursos disponibles en estas unidades, las expectativas de las pacientes y la postura del personal médico tal y como recogen Blanca, Blanco, Luque \& Ramírez (2008) y Mcdermid \& Bagshaw (2009).

La tecnología suele verse como un elemento neutro potenciado por las relaciones sociales, sin embargo, la propia tecnología propicia y es constitutiva de aquellos fenómenos que creemos que son su objeto. Desde esta perspectiva, Hadders (2009b) describe cómo la muerte tiene múltiples actualizaciones en función de la red de actores que la componen. Ninguna de ellas es más auténtica que el resto, aunque sí que podemos considerar que algunas pueden ser más deseables que otras.

\section{La muerte encefálica}

La muerte encefálica, que fue definida en el 1968 por el comité de Ética de Medicina de Harvard, es un ejemplo de las nuevas formas de morir que posibilita el desarrollo tecnológico de los hospitales. Se trata de una conceptualización de la muerte inexistente antes de la introducción de ventiladores mecánicos a mediados del s.XX y de la evolución de las medidas de resucitación (Baron, Shemie, Teitelbaum \& James, 2006). La muerte encefálica se ha definido diferenciándola de la muerte por parada cardiorespiratoria, la cual se caracteriza por la completa interrupción del flujo sanguíneo (parada cardíaca o asistolia) y la cesión consecuente de las funciones vitales (Gherardi, 1997) y estableciendo el coma profundo arreactivo, la ausencia de reflejos troncoencefálicos y la apnea como los pilares del diagnóstico de muerte encefálica (Escudero, 2008). Giacomini (1997) arguye que esta definición, con todo el proceso y todos los actores que moviliza construyó cuerpos en muerte encefálica. En un sentido ligeramente distinto, Trueba (2007) la define como un constructo cultural, y añade que si bien no existe un consenso sobre ésta, se estableció como un hecho tecnocientífico.

Se trata entonces de una forma de morir reciente y que está en controversia (Machado, 1994; Karakatsanis, 2008; Russell, 2000) ya que, compartiendo la conclusión que expone Kaufman (2000), aquellos seres que están mantenidos por prácticas médicas modernas o que no están autoregulándose "naturalmente" y no se los considera ni completamente vivos, ni biológicamente muertos, desestabilizan el orden social existente de modos diferentes respecto otras formas híbridas. En esta línea, Bellomo \& Zamperetti (2007) abordan en un breve texto las implicaciones de asimilar la muerte encefálica a la muerte ya que no se puede decir cuando una persona está muerta, aunque sí que se puede considerar que es un punto de no retorno que puede guiar las decisiones, las prácticas médicas y las normas legales.

Esta complejidad a la hora de definir la muerte la hemos trabajado haciendo un análisis del discurso que construye la muerte encefálica en un manual de coordinación de trasplantes (Flores-Pons \& Íñiguez-Rueda, 2009). Concluimos que la muerte encefálica es construida de formas múltiples: como diagnóstico, como consecuencia, como causa y como proceso y estado. Estas construcciones, aparentemente inconsistentes entre sí, están cumpliendo funciones diferentes en el texto constituyendo la muerte como un objeto factual independiente de la obervadora. En el texto no se aborda en ningún momento la relación entre 'muerte' y 'muerte encefá- 
lica', no obstante, la muerte es un límite no consensuado en muchos ámbitos que, en este caso, al complementarlo con el adjetivo 'encefálica' se despliega como controversia. Con este trabajo abordamos cómo la muerte encefálica se construye como un hecho tecnocientífico, de difícil acceso y difícil cuestionamiento ya que promueve relaciones asimétricas en las que la decisión, en este caso sobre la muerte, queda encapsulada en las prácticas tecnocientíficas biomédicas.

Finalmente, cabe destacar que la muerte encefálica tiene un rol fundamental para el desarrollo del programa de donación y trasplante de órganos y tejidos y que es en este marco que cobra gran parte de su sentido. A partir de estudios etnográficos alrededor de la donación y trasplante de órganos y tejidos (Íñiguez-Rueda, Sanz, Moreno \& Flores-Pons, 2008 Lock, 2002; 2004; Sharp, 2001; 2006) se ha señalado esta complejidad del proceso de diagnóstico de muerte encefálica y la multiplicidad de formas que adquiere así como la diversidad posiciones que genera. Lock (2002) caracteriza la controversia con la expresión twice dead, dos veces muerta. La primera, a partir del acto performativo, en términos de Austin (1962), de declarar la muerte de la persona a partir del diagnóstico de muerte encefálica. Y la segunda, con la parada cardiorespiratoria en el quirófano o en el cubículo de la UCI, según si es donante o no, respectivamente.

\section{Perspectivas}

Asumiendo una posición según la cuál la forma es constitutiva del contenido, consideramos que es relevante hacer un pequeño recorrido por las diferentes perspectivas $\mathrm{y} / \mathrm{o}$ métodos que guían los trabajos sobre la biomedicalización de la muerte. Resulta de extrema ayuda conocer los planteamientos que sustentan los diferentes abordajes posibles de la biomedicalización de la muerte, por este motivo, aunque sin entrar en detalle, a continuación mencionamos algunos de los principales énfasis y perspectivas presentes en estos estudios. Ésta es una clasificación útil porque hace un uso convencional de los términos, hecho que facilita la comprensión rápida que requiere una revisión. No obstante, queremos reivindicar la riqueza y el potencial de romper con las categorías estancas y contemplar las perspectivas como dinámicas e impuras.

Un análisis de las consideraciones que deben tomarse en la reflexión y la actución en el ámbito de la muerte es lo que caracteriza de forma explícita a los estudios en ética. Sin embargo, cabe señalar que todo trabajo tiene una posición ética, así como política, aunque haya una disciplina llamada así propiamente. The ethics of killing. Problems at the margins of life (2002), de Jeff McMahan, es un libro denso pero detallado y analítico que aborda, desde la reflexión ética, gran amplitud de temáticas en las que la muerte se encuentra enmarcada en la biomedicina. De forma más focalizada, Russell (2000) aborda en un libro bastante completo los principales conflictos y conceptos que construyen la muerte encefálica desde una perspectiva de la filosofía y la ética. También sobre la muerte en el marco de la donación de órganos y tejidos trata monográficamente el libro de Miller \& Truog (2011) Death, Dying, and Organ Transplantation: Reconstructing Medical Ethics at the End of Life. Encarado a la práctica biomédica está el trabajo de Lübbe (2003), en el cual analiza los principios éticos de la medicina que deben guiar los cursos de acción del personal médico en la confrontación con la muerte. Trueba (2007), por su parte, dibuja cómo se deben tomar decisiones prácticas asumiendo que la muerte encefálica es un constructo cultural que tiene que responder a las preguntas qué, cómo y cuándo se debe diagnosticar científicamente la muerte y cuál es el grado de evidencia que este diagnóstico asume.

En el estudio de los aspectos legales, podemos destacar el trabajo de Hennette-Vauchez \& Nowenstein (2009) y Nowenstein (2010) como relevantes en el estudio de la articulación entre la legalidad y la biomedicina para la definición de la muerte. Palmer (2000) (revisado por Comfort, 2001) vincula el nacimiento y la muerte, como procesos de tránsito entre estados considerados dicotómicos, para abordar como la ley se integra en la medicina. McLean \& Morgan (2009) por otra parte, estudian los derechos de las pacientes en la regulación legal de la medicina.

El interés por comprender la biomedicalización de la muerte desde la historia es lo que hace que conjuguemos trabajos tan variados en este punto. Resulta imprescindible mencionar el trabajo genealógico de Foucault en El nacimiento de la clínica (1963), un libro que analiza la construcción de la medicina y recoge su relación con la muerte. De un modo muy distinto, Stern (2008) analiza la relación entre la medicina y la muerte históricamente, a partir de tres casos de cuerpos entre la vida y la muerte, dentro de un período histórico de más de siglo y medio. Desde una óptica bastante diferente, Baron et al. (2006) resumen los pasos que ha seguido la definición de muerte encefálica hasta el momento de publicarse su trabajo. Así muestran las controversias que han ido conduciendo, hasta la actualidad, a un consenso mundial bastante amplio en las guías de determinación neurológica de la muerte, pero con bastante disparidad de criterios.

En los estudios de la muerte desde una perspectiva psicosocial, hay un gran número que se pueden considerar así por sus fundamentos anclados en la fenomenología. Son múltiples los trabajos que abordan la biomedicalización de la muerte desde una mirada que inquiere la relación entre la realidad y la experiencia que de ella tienen ciertos actores y la analizan desde una perspectiva ideal, es decir, de cómo esta experiencia se encuentra en el pensamiento, psique o conciencia (Andrew, 1998; Volker \& Limerick, 2007).

Una mirada diferente es aquella que toma el discurso no como mediador, ni como descriptor de la realidad, sino como constituyente de ésta. De este modo, hemos mencionado estudios que abordan la muerte a partir del análisis del discurso que la constituye (Flores-Pons \& Íñiguez-Rueda, 2009; Hadders, 2009a; Li \& Arber, 2006).

Compartiendo importantes antecedentes con el análisis del discurso, el método etnográfico lo utilizó David Sudnow (1967) por primera vez para estudiar la muerte en el hospital, convirtiéndose en el precedente de múltiples estudios entre 
los que cabe destacar Hadders (2007a, 2009b), Lock (2002, 2004), McGibbon \& Peter (2008), Maynard (2006), Sharp (2006).

Por último, la revisión bibliográfica sistemática es otra forma de abordar la muerte y su principal efecto es generar una amplia perspectiva de las formas cómo se ha estudiado. En este sentido, trabajos como los de Allué (1983), Blanca et al., (2008), Kellehear (2008) y Proulx \& Jacelon (2004), facilitan crear una aproximación compleja y permeable a la hora de iniciar una nueva investigación en este ámbito.

\section{Discusión}

La muerte se presenta como un hecho controvertido en la sociedad actual y son múltiples los ámbitos biomédicos en los que se despliega como tal. Se trata de un objeto en disputa tanto en un nivel de definición sobre qué es la muerte, tal y como hemos visto a partir de los trabajos sobre la muerte encefálica, como en un nivel de las implicaciones que debe tener como evento, como sucede a través de las discusiones sobre los cuidados paliativos. Sin embargo, aunque lo veamos como dos debates separados, resulta inconfundible que según cómo se defina qué es la muerte, se definirán los debates que deben acompañarla y viceversa, según cuáles sean las preocupaciones alrededor de la muerte, se delimitará en qué consiste ésta: en qué términos y con qué prácticas se puede definir.

Así, resulta paradójico que la muerte, considerada a menudo como aquello irrevocable e inapelable, un hecho por excelencia, pueda generar tanto debate. Si bien fácilmente se puede concluir que la muerte es invariable y que lo que varía son las formas de verla, de interpretarla o de abordarla, nosotras consideramos que se trata, de hecho, de un objeto múltiple (Mol, 2002). Esto es, aquello que llamamos muerte no es una sola cosa con muchas interpretaciones posibles, sino que es muchas cosas a la vez, cada una de ellas producto de un entramado de actores y de relaciones que la están actuando. Sin embargo, estas múltiples muertes no son independientes, no son objetos distintos sino que están en relación y se configuran como parte de un mismo fenómeno. No hay una única forma de morir, ni una forma natural de morir, sino que cada una de estas muertes es producto de aquello que la está posibilitando. De este modo, aquello que sucede en el hospital, no es una muerte natural en la que la biomedicina interviene, sino que todo el entramado de técnicas, conocimientos, personas, instrumentos, discursos,

\section{Referencias}

Allué, M. (1983). La muerte en Ciencias Sociales: Una bibliografía. Arxiu d'Etnografia de Catalunya, (2). Recuperado a partir de http://antropologia.urv.es/AEC/PDF/N2/Bibliografies/Muerte.pdf

Andrew, C. M. (1998). Optimizing the human experience: nursing the families of people who die in intensive care. Intensive \& Critical Care Nursing: The Official Journal of the British Association of Critical Care Nurses, 14(2), 5965. Recuperado a partir de http://www.ncbi.nlm.nih.gov/pubmed/9814208 afectos o leyes están posibilitando esta muerte. Dicho de otro modo, la muerte es performada, no existe más allá de los agentes que la actualizan, cuando estos agentes dejan de actuar ésta desaparece (ver Latour, 2008 ; Mol, 2002; Tirado \& Domènech, 1998).

Esta perspectiva resulta interesante de cara a potenciar una mirada crítica y reflexiva ya que permite tomar en consideración qué es todo aquello que está actuando para que se produzca una muerte y posibilita pensarla no en términos esencialistas, sino de valor y de voluntad. La muerte no es una esencia a ser descubierta y gestionada cuando emerge, sino que la muerte presenta múltiples opciones de ser en las cuales la decisión tiene cabida. Este planteamiento lleva a poder analizar los diferentes conflictos, controversias y debates que hemos recogido en este artículo desde una mirada que no tome sólo el conflicto que se produce en la situación final, sino también aquello que lo está sustentando. Permite poder preguntarse por la adecuación del sistema en el que se producen la mayoría de las muertes en la sociedad actual, el sistema biomédico. De este modo podemos reivindicar la legitimidad de preguntarnos y decidir sobre qué muerte queremos y ver hasta qué punto el conocimiento y las prácticas biomédicas, con todo lo que las sujeta, están actuando como facilitadoras o dificultadoras de este ejercicio de autodeterminación.

\section{Conclusiones}

Con el objetivo de facilitar el trasvase y el diálogo entre diferentes disciplinas y perspectivas, en este artículo hemos pretendido ofrecer una visión amplia de los trabajos que abordan la biomedicalización de la muerte, así como de las múltiples formas posibles de acercarse a ésta. Este es un paso imprescindible para favorecer un debate y una reflexión desde la complejidad que requiere. La biomedicina se ha erigido como un ámbito de saber de mucha relevancia en las sociedades contemporáneas y su conocimiento ha traspasado desde hace años las paredes de los hospitales y laboratorios, si es que alguna vez estuvieron sólo allí. El imperativo de salud está movilizando y constituyendo estilos de vida, formas de convivencia, formas de ser para sí y para con el resto y la muerte no es sino parte constitutiva de todo ello.

Notas y/o Agradecimientos.- Este trabajo se ha realizado con el apoyo de una beca FPU del Ministerio de Educación y Ciencia (España).

Anspach, R. R. (1987). Prognostic Conflict in Life-and-Death Decisions: The Organization as an Ecology of Knowledge. Journal of Health and Social Behavior, 28(3), 215-231. Recuperado a partir de http://www.jstor.org/stable/2136842

Ariès, P. (1975/2000). Historia de la muerte en Occidente. Desde la Edad Media hasta nuestros días. Barcelona: El Acantilado.

Ariès, P. (1977/1987). El hombre ante la muerte. Madrid: Taurus Ediciones.

Austin, J. L. (1962). Cómo hacer cosas con las palabras. Barcelona: Paidós. 
Banerjee, A. (2008). Disciplining death: hypertension management and the production of mortal subjectivities. Health (London), 12(1), 25-42. doi: $10.1177 / 1363459307083696$

Baron, L., Shemie D., S., Teitelbaum, J., \& James, C. (2006). Brief review: History, concept and controversies in neurologiacal determination of death. Canadian Journal of Anesthesy, 53(6), 602-608.

Bayés, R. (2005). Eutanasia: un dilema ético, médico y jurídico. Cardiología Práctica, 14(1), 32-36.

Bellomo, R., \& Zamperetti, N. (2007). Defining the vital condition for organ donation. Philosophy, Ethics, and Humanities in Medicine, 2(1), 27. doi:10.1186/1747-5341-2-27

Blanca Gutiérrez, J., Blanco Alvariño, A., Luque Pérez, M., \& Ramírez Pérez, M. (2008). Experiencias, percepciones y necesidades en la UCI. Revisión sistemática de estudios cualitativos. Enfermería Global, (12).

Buzzi, E. (2009). "Matters of life and/or death"

Reflections on health care experience: questions of life or cases of death? Journal of Medicine and the Person. Recuperado a partir de http://www.springerlink.com/content/x28808v674548082/fulltext.pdf

Cannaerts, N., de Casterle, B. D., \& Grypdonck, M. (2004). Palliative Care, Care for Life: A Study of the Specificity of Residential Palliative Care. Qual Health Res, 14(6), 816-835. doi:10.1177/1049732304265977

Clarke, A., Mamo, L., \& Fosket, J. R. (2010). Biomedicalization: Technoscience, Health, and Illness in the U.S. Duke University Press.

Comfort, J. (2001). Endings and Beginnings: Law, Medicine, and Society in Assisted Life and Death: Larry I. Palmer. (2000). Westport, CT: Praeger Publishers. 143pp. ISBN 0-275-96681-X. Journal of Government Information, 28(6), 846-848. doi:10.1016/S1352-0237(02)00385-4

Domènech Argemí, M., \& Tirado Serrano, F. (Eds.). (1998). Sociología simétrica. Ensayos sobre ciencia, tecnología y sociedad. Barcelona: Gedisa.

Elias, N. (1982/1987). La soledad de los moribundos (3. ${ }^{\mathrm{a}}$ ed.). Mexico: Fondo de Cultura Económica.

Emanuel, L., Bennett, K., \& Richardson, V. E. (2007). The Dying Role. Journal of Palliative Medicine, 10(1), 159-168. doi:10.1089/ipm.2006.0134

Escudero, D. (2008) La muerte encefálica. Exploración clínica y métodos diagnósticos instrumentales. En R. Matesanz (Ed.) El modelo español de trasplante de órganos y tejidos (2 ${ }^{a}$ ed.) pp.121-134. Madrid: Aula Médica.

Flores-Pons, G., \& Íñiguez-Rueda, L. (2009). Análisis del discurso tecnocientífico que construye la muerte encefálica en un manual de coordinación de trasplantes. Discurso \& Sociedad, 3(4), 682-713. Recuperado a partir http://www.dissoc.org/ediciones/v03n04/DS3\%284\%29Flores\&Inigu ez.pdf

Flores-Pons, G., Moreno, E., Íñiguez-Rueda, L. \& Sanz, J. Anticipación en biomedicina: la transferencia d eórganos y tejidos.(en prensa)

Foucault, M. (1963/1975). El nacimiento de la clínica (6. ${ }^{\mathrm{a}}$ ed.). Mexico: Siglo XXI Editores.

Foucault, M. (1978/1980). Microfisica del poder (2. ${ }^{\mathrm{a}}$ ed.). Madrid: Ediciones la Piqueta.

Friedman, R., \& James, J. W. (2008). The Myth of the Stages of Dying, Death and Grief. Skeptic, 14(2), 37-41.

Gascón Abellán, M. (2003). ¿De qué estamos hablando cuando hablamos de eutanasia? Humanitas, Humanidades Médicas, 1(1), 15-22

Gherardi, C. R. (1997). La muerte cerebral y la muerte. Medicina Buenos Aires, 57, 114-118. Recuperado a partir de http://www.medicinabuenosaires.com/revistas/vol5797/1/ muertecereb.htm

Giacomini, M. (1997). A change of heart and a change of mind? Technology and the redefinition of death in 1968. Social Science \& Medicine, 44(10), 1465-1482. doi:10.1016/S0277-9536(96)00266-3

Hadders, H. (2007a). Relatives' presence in connection with cardiopulmonary resuscitation and sudden death at the intensive care unit. Nursing Inquiry, 14(3), 224-232. doi:10.1111/j.1440-1800.2007.00371.x

Hadders, H. (2007b). Dealing with the dead patient at the intensive care unit. Mortality, 12(3).

Hadders, H. (2009a). Medical practice, procedure manuals and the standardisation of hospital death. Nursing Inquiry, 16(1), 22-32.
Hadders, H. (2009b). Enacting death in the intensive care unit: medical technology and the multiple ontologies of death. Health (London), 13(6), 571-587. doi:10.1177/1363459308341869

Hart, B., Sainsbury, P., \& Short, S. (1998). Whose dying? A sociological critique of the 'good death'. Mortality: Promoting the interdisciplinary study of death and dying, 3(1), 65. doi:10.1080/713685884

Harvey, J. (1997). The Technological Regulation of Death: With Reference to the Technological Regulation of Birth. Sociology, 31(4), 719-735. doi: $10.1177 / 0038038597031004005$

Hennette-Vauchez S. \& Nowenstein, G. (2009). «Dire la mort et faire mourir » Tensions autour de la mortencéphalique et la fin de vie en france, Sociétés contemporaines, 75 (3), p. 37-57. : 10.3917/soco.075.0037

Heyland, D. K., Tranmer, J., \& Feldman-Stewart, D. (2000). End-of-life decision making in the seriously ill hospitalized patient: an organizing framework and results of a preliminary study. Journal of Palliative Care, 16 Suppl, S31-39. Recuperado a partir de http://www.ncbi.nlm.nih.gov/pubmed/11075531

Holstein, M. (1997). Reflections on death and dying. Academic Medicine: Journal of the Association of American Medical Colleges, 72(10), 848-855. Recuperado a partir de http://www.ncbi.nlm.nih.gov/pubmed/9347706

Iñiguez-Rueda, L., Sanz Porras, J., Moreno Gabriel, E, \& Flores-Pons, G. (2008) Estudi etnográfic dels moments clau en l'itinerari de donació d'òrgans $i$ teixits. Fonaments qualitatius per a una millora de les pràctiques hospitalàries. Premi Caixa Sabadell. Documento no publicado.

Jerez Juan, Montserrat \& Rodríguez Díaz, José A. (1994). El cuerpo humano ante las nuevas tecnologías médicas Hacia una redefinición del nacimiento y la muerte. Revista Española de Investigaciones Sociológicas, (68). Recuperado partir http://www.reis.cis.es/REISWeb/PDF/REIS 068 10.PDF

Karakatsanis, K. (2008). Brain death: Should it be reconsidered? Spinal Cord, 46, 396-401.

Kaufman, S. R. (2000). In the Shadow of "Death with Dignity": Medicine and Cultural Quandaries of the Vegetative State. American Anthropologist, New Series, 102(1), 69-83. Recuperado a partir de http://www.jstor.org/stable/683539

Keating, P., \& Cambrosio, A. (2000). Biomedical platforms. Configurations, 8(3), 337-387.

Kellehear, A. (2008). Dying as a social relationship: A sociological review of debates on the determination of death. Social Science \& Medicine, 66(7), 1533-1544. doi:10.1016/i.socscimed.2007.12.023

Kübler-Ross, E. (1969/2009) On Death and Dying, Routledge: Oxon.

Latour, B. (1987/1992). Ciencia en acción (2. ' ed.). Barcelona: Labor.

Latour, B. (2008). Reensamblar lo social. Una introducción a la teoría del actor-red (1. ${ }^{\text {a }}$ ed.). Buenos Aires: Manantial.

Li, S., \& Arber, A. (2006). The Cnstruction of Troubled and Credible Patients: A Study of Emotion Talk in Palliative Care Settings. Qual Health Res, 16(1), 27-46. doi:10.1177/1049732305284022

Lock, M. (2002). Twice dead: Organs donation and the reinvention of death. California: California Press.

Lock, M. (2004). Living Cadavers and the Calculation of Death. Body Society, 10(2-3), 135-152. doi:10.1177/1357034X04042940

Long, S. O. (2004). Cultural scripts for a good death in Japan and the United States: similarities and differences. Social Science \& Medicine, 58(5), 913-928. doi:10.1016/i.socscimed.2003.10.037

Lübbe, A. (2003). No public health without public death-ethical considerations. Supportive Care in Cancer, 11(8), 497-500. Recuperado a partir de http://www.springerlink.com/content/vcaphaav2ig1be08/fulltext.pdf

Machado, C. (1994). Death on neurological grounds. Journal on Neurosurgical Science, 38(4), 209-222.

Maynard, R. J. (2006). Controlling Death: Compromising Life: Chronic Disease, Prognostication, and the New Biotechnologies. Medical Anthropology Quarterly, New Series, 20(2), 212-234. Recuperado a partir de http://www.jstor.org/stable/3655413

McClement, S. E., Fallis, W. M., \& Pereira, A. (2009). Family Presence During Resuscitation: Canadian Critical Care Nurses' Perspectives. Journal of Nursing Scholarship, 41(3), 233-240. doi:10.1111/i.15475069.2009.01288.x

McDermid, R., \& Bagshaw, S. (2009). Prolonging life and delaying death: The role of physicians in the context of limited intensive care resources. 
Philosophy, Ethics, and Humanities in Medicine, 4(1), 3. doi:10.1186/1747$\underline{5341-4-3}$

McGahey-Oakland, P. R., Lieder, H. S., Young, A., \& Jefferson, L. S. (2007). Family Experiences During Resuscitation at a Children's Hospital Emergency Department. Journal of Pediatric Health Care, 21(4), $217-$ 225. doi:10.1016/i.pedhc.2006.12.001

McGibbon, E., \& Peter, E. (2008). An Ethnography of Everyday Caring for the Living, the Dying, and the Dead: Toward a Biomedical Technography. Qualitative Inquiry, 14(7), 1134-1156. doi:10.1177/1077800408322229

McLean, S. A. M., \& Morgan, D. (2009). CHOOSING DEATH OR LIFE: LAW, MEDICINE AND PATIENTS' RIGHTS. Juridical Review, 2009(2), 129-148. Recuperado a partir de http://search.ebscohost.com/login.aspx?direct $=$ true\&db=tnh\&AN=4 4141914/=es\&site=ehost-live

McNamara, B., Waddell, C., \& Colvin, M. (1994). The institutionalization of the good death. Social Science \& Medicine, 39(11), 1501-1508. doi:10.1016/0277-9536(94)90002-7

McMahan, J. (2002). The ethics of killing. Problems at the margins of life. New York: Oxford University Press.

McPherson, C. J., Wilson, K. G., \& Murray, M. A. (2007). Feeling like a burden to others: a systematic review focusing on the end of life. Palliative Medicine, 21(2), 115-128. doi:10.1177/0269216307076345

Menezes Aisengart, R. (2008). Cuidados Paliativos: caceptación social de la muerte? Cultura y Religión. Recuperado a partir de http://www.revistaculturayreligion.cl/articulos/vol2_n1_2008_junio_0 8 rachel_aisengart_meneses.pdf

Miller, F. G., \& Truog, R. (2011). Death, Dying, and Organ Transplantation: Reconstructing Medical Ethics at the End of Life. New York: Oxford University Press.

Mol, A.M. (2002). The body multiple. , SC, and London: Duke University Press

Muñoz Justicia, Juan (2005). Análisis de datos textuales con Atlas.ti 5. Recuperado a partir de http://psicologiasocial.uab.es/juan/index.php?option=com_docman\& task $=$ doc download \&gid $=1 \&$ Itemid $=$

Muñoz-Justicia, J., \& Sahagún-Padilla, M. Á. (2011). Análisis cualitativo asistido por ordenador con ATLAS.ti. In C. Izquierdo \& A. Perinat (Eds.), Investigar en Psicología de la Educación. Nuevas Perspectivas Conceptuales y Metodológicas (pp. 299-363). Barcelona: Amentia. Recuperado a partir de

http://psicologiasocial.uab.es/juan/index.php?option=com_custompr operties\&task $=\operatorname{tag} \& \operatorname{tag} N a m e=$ Publicaciones $\% 3$ AMis + textos\&Itemid $=$ $\underline{101}$

Nowenstein, G. (2010). The Generosity of the Dead: A Sociology of Organ Procurement in France. Ashgate Publishing, Ltd.

Olshansky, S. J., Rudberg, M. A., Carnes, B. A., Cassel, C. K., \& Brody, J. A. (1991). Trading Off Longer Life for Worsening Health: The Expansion of Morbidity Hypothesis. J Aging Health, 3(2), 194-216. doi: $10.1177 / 089826439100300205$

Palmer, L. I. (2000) Endings and Beginnings: Law, Medicine, and Society in Assisted Life and Death, CT: Praeger Publishers.

Proulx, K., \& Jacelon, C. (2004). Dying with dignity: The good patient versus the good death. American Journal of Hospice and Palliative Medicine, 21(2), 116-120. doi:10.1177/104990910402100209

Rose, N. (2007). The politics of life itself. Biomedicine, power, and subjectivity in the twenty-first century. New Jersey: Princeton University Press.
Rosenfeld, B. (2004). Assisted Suicide and the right to die. The interface of social science, public policy, and medical ethics. Washington DC: American Psychological Association.

Rovaletti, ML (2002). La ambigüedad de la muerte: reflexiones entorno a la muerte contemporánea. Revista Colombiana de Psiquiatría, 31 (2), 91 108. Recuperado a partir de http://www.scielo.org.co/pdf/rcp/v31n2/v31n2a04.pdf

Russell, T. (2000). Brain death. Philosophical concepts and problems. Hampshire: Ashgate.

Seymour, J. E. (2000). Negotiating natural death in intensive care. Social Science \& Medicine, 51(8), 1241-1252. doi:10.1016/S0277-9536(00)00042-3

Sharp, L. A. (2001). Commodified Kin: Death, Mourning, and Competing Claims on the Bodies of Organ Donors in the United States. American Anthropologist, New Series, 103(1), 112-133. Recuperado a partir de http://www.jstor.org/stable/683925

Sharp, L. A. (2006). Strange Harvest. Organ Transplants, Denatured Bodies, and the Transformed Self. Berkeley: University of California Press.

Stern, M. (2008). [']Yes:--no:--I have been sleeping--and now--now--I am dead': undeath, the body and medicine. Studies in History and Philosophy of Science Part C: Studies in History and Philosophy of Biological and Biomedical Sciences, 39(3), 347-354. doi:10.1016/i.shpsc.2008.06.008

Sudnow, D. (1967). La organización social de la muerte (1971). Buenos Aires: Editorial Tiempo Contemporáneo.

Taylor, B., Glass, N., McFarlane, J., \& Stirling, C. (1997). Palliative nurses' perceptions of the nature and of their work. International Journal of Palliative Nursing, 3(5), 253-258.

Thomas, L.-V. (1975/1993). Antropología de la Muerte. Fondo de Cultura Económica.

Thomas, L.-V. (1991). La muerte. Barcelona: Paidós.

Timmermans, S. (1998). Resuscitation Technology in the Emergency Department: Towards a Dignified Death. Sociology of Health \& Illness, 20, 144-167. Recuperado a partir de http://www.ingentaconnect.com/content/bpl/shil/1998/00000020/0 $\underline{0000002 / \operatorname{art} 00095}$

Timmermans, S. \& Berg, M. (1997). Standardization in Action: Achieving Local Universality through Medical Protocols. Social Studies of Science, 27(2), 273-305.

Trueba Gutiérrez, J. L. (2007). La muerte clínica: un diagnóstico y un testimonio. Anales del sistema sanitario de Navarra, 30(Extra 3), 57-70.

Turner, L. (2004). Life Extension Research: Health, Illness, and Death. Health Care Analysis, 12(2). Recuperado a partir de http://www.springerlink.com/content/gpnh252v3383578g/fulltext.pdf

Volker, D. L. (2001). Oncology nurses' experiences with requests for assisted dying from terminally ill patients with cancer. Oncology Nursing Forum, 28(1), 39-49. Recuperado a partir de http://www.ncbi.nlm.nih.gov/pubmed/11198896

Volker, D. L., \& Limerick, M. (2007). What constitutes a dignified death? The voice of oncology advanced practice nurses. Clinical Nurse Specialist CNS, 21(5), 241-247; quiz 248-249. doi:10.1097/01.NUR.0000289749.77866.7c

Woolgar, S. (1988/1991). Ciencia. Abriendo la caja negra. Barcelona: Anthropos.

Zimmermann, C., \& Rodin, G. (2004). The denial of death thesis: sociological critique and implications for palliative care. Palliative Medicine, 18(2), 121-128. doi:10.1191/0269216304pm858oa

(Artículo recibido: 31-10-2010, revisado: 12-4-2012, aceptado: 21-4-2012 